\title{
Cuerpo, self y sociedad: una reflexión desde la fenomenología y el interaccionismo simbólico
}

Body, self, and society: a reflection from phenomenology and symbolic interactionism approaches

\section{Itzel Adriana Sosa-Sánchez*}

itzela@crim.unam.mx

ORCID: https://orcid.org/0000-0002-1223-0829

Doctora en Sociología

Centro Regional de Investigaciones Multidisciplinarias de la Universidad Nacional Autónoma de México

México

\section{Resumen}

Con la finalidad de ahondar en la comprensión de los vínculos entre el cuerpo, la sociedad y el self, en este ensayo se presenta una reflexión sobre las aportaciones teóricas y conceptuales del interaccionismo simbólico, de la sociología fenomenológica y de la fenomenología de Merleau-Ponty. En los abordajes analizados, podemos encontrar conceptualizaciones que hacen referencia a la imposibilidad de pensar el self y el cuerpo fuera de la historia. El cuerpo, en tanto objeto de estudio sociológico, puede (y debe) ser

* Es investigadora en el Centro Regional de Investigaciones Multidisciplinarias de la Universidad Nacional Autónoma de México (CRIM-UNAM), adscrita al programa de Estudios de Población. Es doctora en Sociología por la Universidad Laval (Québec, Canadá). Sus áreas de interés incluyen los estudios de género y el análisis interseccional, el embarazo adolescente, la sociología del cuerpo, la sociología de las relaciones inter-étnicas, la salud sexual y reproductiva, la sociología de la sexualidad, los derechos sexuales y reproductivos, las metodologías cualitativas y cuantitativas. Es miembro del Sistema Nacional de Investigadores (SNI).

Sosa-Sánchez, I. A. (2021). Cuerpo, self y sociedad: una reflexión desde la fenomenología y el interaccionismo simbólico. Iberoforum. Revista de Ciencias Sociales, Nueva Época, 1(2), 1-18, Ensayos, e000162. https://doi.org/10.48102/if.2021.v1.n2.162 Licencia Pública Internacional - CC BY-NC-ND 4.0 
pensado como un nudo de estructura y acción a través del cual es posible acceder al análisis de los vínculos y las relaciones entre el sujeto, el cuerpo y la sociedad, asumiendo que la acción social es incorporada necesariamente. Al mismo tiempo, los vínculos entre cuerpo, self y sociedad obligan a repensar los vínculos entre lo macro y lo micro social y a cuestionar el pensamiento binario que ha predominado en las ciencias sociales.

\section{Palabras clave}

Self, cuerpo, sociedad, interaccionismo simbólico, fenomenología.

\section{Abstract}

To expand the understanding of the links between the body, society and the self, this essay introduces a reflection on the theoretical and conceptual contributions of symbolic interactionism, phenomenological sociology and Merleau-Ponty's phenomenology. In the explored approaches, we can find conceptualizations that refer to the impossibility of thinking the self and the body outside history. The body, as an object of sociological study, can (and must) be thought of as a knot of structure and action through which it is possible to access the analysis of the ties and relationships between the subject, the body and society, assuming that social action is necessarily integrated. At the same time, the links between body, self and society force us to rethink the links between the macro and the micro social and to question the binary thinking that has predominated in social sciences.

\section{Keywords}

Self, body, society, symbolic interactionism, phenomenology.

\section{Introducción}

La reciente producción en ciencias sociales sobre el cuerpo ha retomado diversas tradiciones que lo han abordado a través del tiempo. Bajo esta línea, en este ensayo, se presenta una reflexión sobre los vínculos entre el cuerpo, la sociedad y el self, retomando los fundamentos teóricos y conceptuales del interaccionismo, ${ }^{1}$ de la sociología fenomenológica y de la fenomenología

1 Pese a que se tiende a pensar al interaccionismo como una escuela homogénea, existen diversas aproximaciones desarrolladas bajo la misma. Se ha sugerido que existen al menos dos aproximaciones interaccionistas ancladas en diferentes tradiciones intelectuales: la ba-

Sosa-Sánchez, I. A. (2021). Cuerpo, self y sociedad: una reflexión desde la fenomenología y el interaccionismo simbólico. Iberoforum. Revista de Ciencias Sociales, Nueva Época, 1(2), 1-18, Ensayos, e000162. https://doi.org/10.48102/if.2021.v1.n2.162 Licencia Pública Internacional - CC BY-NC-ND 4.0 
de Merleau-Ponty con el fin de visibilizar las principales aportaciones de éstos a los debates teórico-metodológicos en las ciencias sociales; asimismo, se destaca el peso de las corrientes abordadas en los debates posteriores sobre el cuerpo que han surgido en las ciencias sociales.

\section{El self y el mundo social en el interaccionismo simbólico}

A grandes rasgos, el self en ciencias sociales suele referir "al proyecto simbólico que permite orientar al individuo en su relación consigo mismo, con otros y con las cosas" (Bernasconi, 2015, p. 2). Igualmente, este concepto "alude al ejercicio auto-reflexivo mediante el cual el individuo se interpela a sí mismo, intentado responder en primera persona a la pregunta por su identidad" (Bernasconi, 2015, p. 3). El self, como concepto, desde el interaccionismo social, es, ante todo, un proceso social que alude al carácter contingente y dinámico de la acción social negociada. Para el interaccionismo, el self es una piedra angular de su aparato conceptual -y en el caso de Goffman (1974), es una unidad de análisis fundamental-. Pese a que hay diferentes definiciones del self, existen algunos rasgos comunes compartidos dentro del interaccionismo simbólico. Desde esta perspectiva, el self emana de procesos sociales diversos y de la experiencia adquirida, siendo una instancia de reflexión y reflexividad, un ordenador de situaciones y una fuente de sentido que rige la relación del individuo con el mundo. Se asume que el self es una modalidad de la conciencia que orienta los hechos, los gestos y las acciones hacia otros, por lo que un self sin otros es impensable.

A su vez, el concepto del self está estrechamente ligado al concepto del otro generalizado, que, desde el interaccionismo, hace referencia a cómo las personas actúan en referencia no sólo al otro inmediato, sino en relación con un otro social generalizado (resultante de los procesos de socialización) que representa el conjunto de actitudes y perspectivas a asumir según el grupo de adscripción (Mead, 1962). El interaccionismo considera la sociedad como una estructura dinámica de la vida cotidiana, construida por los actores sociales, en donde la interacción involucra no sólo a éstos, sino a una multitud de otros invisibles que impregnan la relación de los actores con el mundo en dicha interacción (Le Breton, 2004).

sada en el trabajo de William Thomas y Robert Park y la basada en el interaccionismo de Herbert Mead, que fue posteriormente retrabajada y codificada por Herbert Blumer. Para efectos de este ensayo, nos centraremos en la escuela derivada de las reflexiones de Mead.

Sosa-Sánchez, I. A. (2021). Cuerpo, self y sociedad: una reflexión desde la fenomenología y el interaccionismo simbólico. Iberoforum. Revista de Ciencias Sociales, Nueva Época, 1(2), 1-18, Ensayos, e000162. https://doi.org/10.48102/if.2021.v1.n2.162 Licencia Pública Internacional - CC BY-NC-ND 4.0 
Una de las principales cuestiones concernientes al self es dónde se ubica. Si bien este concepto está claramente vinculado al individuo, es asumido como una entidad social tanto por Mead y Blumer como por Goffman. Por ejemplo, Mead (1962) refiere que el self es donde radica la conciencia y resulta de la experiencia social, la cual, a su vez, es el resultado de la interacción y de una conciencia también social. Mead enfatizó la naturaleza social de la organización de los significados, lo que cambió las conceptualizaciones que, hasta ese momento, existían sobre el self, la mente y la sociedad, puesto que este autor los caracterizó como procesos emergentes y activos de las interacciones sociales (Allan, 2007). Para Mead (1962), el self, como todos los objetos sociales, debe denotar y dotar de significado a través de las interacciones sociales, es decir, es un proceso. ${ }^{2}$

Por su parte, Goffman (1973 y 1974), interesado especialmente en la interacción cara a cara, resalta la influencia recíproca de los individuos sobre las acciones de los otros cuando están ante la presencia física del otro. Goffman (1973) considera que la vida es un escenario con actores y espectadores. En ese escenario, el actor presenta el self, definiendo las situaciones con objeto de controlar la impresión de los otros (espectadores) (Roberts, 2006). Nizet y Rigaux (2005), siguiendo la aproximación goffmaniana, identifican tres componentes del self en la interacción: el primero lo constituye el juego (en un sentido lúdico y teatral) y sus interpretaciones; así, el self es producido a través del compromiso de los actores en la interacción en un sentido dialéctico, en tanto que el self se encuentra con el mundo y viceversa. Esto es, el self es producido por los actos verbales y no verbales del actor social, y resulta de las interpretaciones que éste hace de los actos de los otros, asumiendo que el self es algo que se actúa. El self no es solamente el resultado del rol jugado por el individuo, sino que se construye en relación con la definición que otros tengan del rol que se debe asumir en determinadas situaciones.

El segundo componente es la sacralización de los rostros individuales, una de las características centrales del orden social mediante la cual se atribuyen valores a los individuos que participan en el juego. El tercer

2 Fue para enfatizar el carácter social de los gestos que Mead elaboró este concepto. El autor resaltó el significado de la interacción individual, denominándola "conversación de gestos", asumiendo éstos como símbolos significantes socialmente compartidos por los individuos que pertenecen a una sociedad y a un cierto grupo social. Para Mead (1962), los gestos hacen posible la "simbolización de la experiencia" dentro del campo más amplio de significados. 
componente lo constituyen los fundamentos ceremoniales del self, que se adecúan también según las circunstancias. En elaboraciones posteriores de Goffman sobre el self, éste aparece como un proceso social; esto implica asumir que el self es más que un conjunto de roles: es una "fórmula" organizadora que produce los medios para gestionar la relación entre los deseos y las expectativas de roles específicos (Roberts, 2006). Goffman también define el self como enteramente social, aunque argumenta que existe un componente no socializado del mismo (Roberts, 2006). Al mismo tiempo, Goffman coloca el self al centro del orden de la interacción: no en el individuo, sino en el encuentro social, ${ }^{3}$ siendo el self un proceso social, una entidad dinámica y fluida que es continuamente transformada por su posesor, anclado en la biografía individual del actor social antes y después de cada evento social; asimismo, es el producto de todas las disposiciones escénicas de los actores sociales (Goffman, 1973).

\section{La sociología fenomenológica de Schütz}

Podemos afirmar que una de las bases principales del proyecto filosófico-sociológico de Schütz y del proyecto fenomenológico de Merleau-Ponty es la fenomenología de Husserl. La palabra fenomenología significa "estudio del fenómeno", en donde la noción de fenómeno puede aproximarse a la noción de experiencia; ${ }^{4}$ la fenomenología se centra sobre todo en el carácter y en la estructura de la misma (Cerbone, 2006), y propone trascender la cultura y reducir el fenómeno en cuestión a su esencia subjetiva (Allan, 2007; Arias, 1975).

Se considera a Husserl como su fundador; dos de sus temas principales (siguiendo a Brentano) fueron las nociones de intencionalidad y conciencia; Husserl se centra en desentrañar cómo experimentamos los fenómenos y en qué representan éstos para la conciencia (Cerbone, 2006). Para la fenomenología, la intencionalidad (la operación mental que se dirige hacia los objetos) se refiere a las propiedades de la conciencia o a la relación conciente que se tiene con los objetos (intencionales) y en cuya

3 Asumiendo que en cada encuentro el individuo adquiere un rol situacional que se acompaña de un self situado.

4 La fenomenología está particularmente interesada en la manera en la que las cosas se muestran o aparecen ante nosotros. Sin embargo, si bien muchas veces en las disertaciones fenomenológicas aparece la percepción, ésta es sólo una de las manifestaciones de dicha experiencia. 
constitución participan significados. La intencionalidad requiere la síntesis de significados y actos de conciencia (recordar, contemplar, soñar, etcétera) y, por lo tanto, de intersubjetividad; ésta alude a la percepción que se tiene de que experimentamos el mundo de manera sobreentendida y compartida por otros (Allan, 2007).

En lo que respecta a las reflexiones fenomenológicas de Schütz, este autor planteó una refundación metodológica en ciencias sociales bajo la forma de una sociología fenomenológica o una sociología general de lo cotidiano con consecuencias sobre la investigación empírica en ciencias sociales (Schütz, 1987 y 1962). Para Schütz, el objeto principal de las ciencias sociales empíricas no es la fenomenología trascendental, sino la fenomenología constitutiva de la actitud natural; de allí que señale que la principal aportación de Husserl a las ciencias sociales es justamente su análisis sobre los problemas del mundo de la vida (Lebenswelt) (Schütz, 1998 y 1987). ${ }^{5}$ Schütz define el mundo de la vida, siguiendo a Husserl, como la estructura en la que, al asumir una actitud natural, los humanos experimentan la cultura y la sociedad, y en la que se asumen posturas ante los objetos que influyen en nuestro modo de actuar (Schütz, 1998 y 1962). Siguiendo a Husserl, Schütz dirige su atención hacia "el mundo del sentido común" (el cual es, de inicio, un mundo preinterpretado) (Schütz, 1962).

Schütz converge con autores interaccionistas al mostrar un marcado interés en la definición de situaciones sociales, la construcción de las interacciones (Schütz, 1962) y de la experiencia. Los análisis de Schütz se centraron en la vida cotidiana, con la asunción de que el mundo del sentido común está condicionado culturalmente ${ }^{6}$ y está lleno de significados socialmente producidos (intersubjetividad), ${ }^{7}$ siendo este mundo, por excelencia, el área de la acción social (Schütz, 1962 y 1998).

El análisis fenomenológico de la experiencia de la vida cotidiana se abstiene de adscribirse a hipótesis causales, genéticas o esencialistas sobre

5 Para Husserl, los supuestos corrientes del mundo de la vida (Lebenswelt) deben eliminarse para revelar la "subjetividad pura", ya que aprehendiendo ésta podemos conocer el mundo en sus esencias.

6 Para Schütz (1987 y 1998), la cultura puede ser definida en términos de los conocimientos compartidos, socialmente producidos y aprobados.

7 Respecto a la producción de significados, para Schütz (1987 y 1998), la intencionalidad es la base de la conciencia y del significado, considerando que los significados se relacionan con la conciencia individual y con procesos individuales y subjetivos. 
el mundo de la vida. Desde esta perspectiva, "lo social" designa una relación entre dos o más personas que interactúan entre sí y producen motivos subjetivos que orientan la acción social; aquí, la noción de acción social alude a la noción de sentido (Blin, 1998). El objeto de una sociología fenomenológica está dado por la suma total de los objetos y sucesos del mundo social y cultural tal y como son experimentados desde el "sentido común" de la vida cotidiana y desde las relaciones de interacción establecidas entre dichos objetos (Schütz, 1987 y 1998). A su vez, estas relaciones de interacción constitutivas de la realidad social pueden ser entendidas en relación con las estructuras subjetivas de la percepción, es decir, en las estructuras de la conciencia subjetivas, mediante las cuales se experimenta el mundo de la vida cotidiana.

En cuanto a la estructuración de este mundo (y la experiencia social del mismo), podemos mencionar que éste se estructura de manera espacio-temporal y está marcado por la contemporaneidad (Schütz, 1998). ${ }^{8}$ Una de las preocupaciones centrales para Schütz fue la experiencia de las personas sobre la vida cotidiana como real, a través de una actitud natural, que es un mecanismo mediante el cual el mundo de la vida (y su permanencia) es asumido como "real" y como dado. Si bien la realidad del sentido común conforma la matriz de toda acción social, Schütz reconoce que cada individuo se ubica en la vida diaria de un modo específico, a partir de elementos aportados por lo que denomina la "situación biográfica".

Esta última dota al individuo de lo que Schütz denomina el stock de conocimientos disponibles (stock of knowledge at hand) que poseen los individuos en cualquier momento de la vida; dicho stock juega un rol central en el pensamiento del sentido común (Schütz, 1962). ${ }^{9}$ Este stock está constituido por tipificaciones del mundo del sentido común; es moldeado por la experiencia y, como toda forma de conocimiento, está socialmente enraizado, distribuido y transmitido, siendo el vocabulario y la sintaxis del lenguaje el medio tipificador del conocimiento social por excelencia (Schütz, 1962). El stock de conocimientos efectivos no es otra cosa que la sedimen-

8 La contemporaneidad implica la coexistencia temporal con otros sujetos, incluso si éstos no se hallan en una proximidad espacial recíproca (Schütz, 1998).

9 Para Schütz (1962), incluso el conocimiento de las diferencias individuales de conocimientos es en sí mismo un elemento de la experiencia del sentido común (por ejemplo, cuando una persona lega reconoce su diferencia de conocimientos respecto a la salud y busca ayuda médica).

Sosa-Sánchez, I. A. (2021). Cuerpo, self y sociedad: una reflexión desde la fenomenología y el interaccionismo simbólico. Iberoforum. Revista de Ciencias Sociales, Nueva Época, 1(2), 1-18, Ensayos, e000162. https://doi.org/10.48102/if.2021.v1.n2.162 Licencia Pública Internacional - CC BY-NC-ND 4.0 
tación de todas las experiencias que se tienen sobre las definiciones de situaciones pasadas, experiencias que se refieren a nuestro mundo (Schütz, 1998 y 1962). La expresión individualizada de este stock está en función del lugar que ocupa el individuo en el mundo social (Schütz, 1962). Así, la situación biográfica ${ }^{10}$ define la manera en la que los individuos se ubican en la arena de la acción social, interpretando sus posibilidades y limitaciones (Schütz, 1962).

Por otro lado, Schütz elaboró una teoría interpretativa influenciada también por el "individualizado" Verstehen weberiano, enfatizando las diferencias entre significados y acciones, y entre actos y acciones (Schütz, 1962 y 1987; Wanderer, 2005). Desde esta perspectiva, el término acción designa la conducta humana como un proceso continuo que es planeado por los actores con anticipación y se basa en un proyecto preconcebido. El acto designa el resultado de este proceso continuo, la acción una vez llevada a cabo (Schütz, 1962).

Pese a las múltiples críticas de Weber elaboradas por Schütz, este autor retoma el esquema de la sociología comprensiva weberiana y distingue tres niveles de la Verstehen: 1) en tanto forma de experiencia del conocimiento común cotidiano, 2) en tanto problema epistemológico y 3) en tanto método específico de las ciencias sociales (Schütz, 1962 y 1998; Blin, 1998 y 2007). Como forma de experiencia del sentido común, Verstehen significa para Schütz que los seres humanos interpretan en su vida diaria el mundo de manera significativa (meaningful). Como problema epistemológico, Verstehen se enraíza en lo que Husserl denominó Lebenswelt (el mundo de la vida), englobando la totalidad y riqueza de la experiencia del sentido común vivida por los individuos desde una existencia concreta.

El Lebenswelt también constituye la base para comprender el significado de Verstehen como método específico de las ciencias sociales. ${ }^{11} \mathrm{Se}$ considera aquí que los científicos sociales forman parte de la realidad que pretenden estudiar, lo que hace de la ciencia social una ciencia que elabora

10 En Schütz (1998), el concepto de situación consta de dos componentes: el primero encuentra sus orígenes en la estructura ontológica del mundo dado; el segundo proviene del estado biográfico actual del individuo e incluye su stock de conocimientos en su articulación efectiva.

11 Bajo el entendido de que las ciencias humanas producen sentido sobre sí mismas, sobre su propio sentido. Blin (1998) señala que las preocupaciones empíricas son las que llevan a Schütz a orientarse hacia el sentido subjetivo. 
construcciones de segundo grado, ${ }^{12}$ en donde Verstehen es empleado para expresar la realidad subjetiva de los actores sociales a la cual se busca acceder y comprender (Schütz, 1962).

Igualmente, Schütz asume que el self de nuestra experiencia no es un self estrictamente individual, sino que es producido socialmente. Schütz (1962 y 1987) considera que se adquiere conciencia del mismo y de las experiencias a través del lenguaje. Así, el mundo de la vida es lingüísticamente construido y experimentado a través de una matriz de relaciones en donde el self y los otros están estrechamente unidos. De esta manera, el mundo de la vida es experimentado como intersubjetivo a través de un mecanismo denominado "reciprocidad de perspectivas", que se basa en dos supuestos: a) que se actúa considerando que existe un sólo mundo objetivo y accesible para todos (mundo compartido) y b) que las personas son pragmáticamente motivadas para actuar en cada situación, según preocupaciones prácticas (Schütz, 1962 y 1987).

\section{Aportaciones del interaccionismo simbólico y de la fenomenología de Merleau-Ponty en torno al cuerpo}

Podemos afirmar que el estudio del cuerpo, como un objeto central en las ciencias sociales (lo que ha tendido a denominarse la teoría social del cuerpo), es un enfoque teórico-metodológico relativamente nuevo que supone un distanciamiento respecto a abordajes anteriores sobre el cuerpo (Esteban, 2004; Adelman y Ruggi, 2013). En el interaccionismo simbólico, puede considerarse que fue Mead quien confirió a las actitudes corporales un significado preciso, pues fue el primero en examinar lo que se denomina la simbólica corporal. Desde esta perspectiva, se asume que los gestos, las palabras y las actitudes de un individuo son recibidos por los otros como símbolos, es decir, como portadores de significados a los cuales se deben ajustar para construir una respuesta adecuada y situacional frente a los mismos (Le Breton, 2004).

12 Lo que no significa que estén fuera de las acciones y significados del sentido común, que son construcciones de primer orden, lo que alude a la existencia de múltiples realidades (Roberts, 2006). 
Por su parte, Goffman colocó el cuerpo en el centro de sus análisis sociológicos sobre el orden en la interacción. ${ }^{13}$ Goffman demostró que la habilidad de intervenir en el mundo social está en función del manejo que los actores sociales tienen de sus cuerpos y de cómo éstos se orientan a partir, por y hacia otros cuerpos en el tiempo y el espacio (Shilling, $2004 \mathrm{y}$ 2005). Goffman (1973 y 1974) se centró en los procesos de interacción social para explorar los diferentes registros cognitivos, sensoriales, afectivos y corporales de la acción, por lo que el análisis sociológico -desde esta perspectiva - focaliza la mirada sociológica sobre el cuerpo y muestra las diferentes relaciones establecidas en la experiencia de los actores sociales.

Así, para Goffman (1963), el cuerpo emerge en ciertas situaciones como eje central en la confrontación de las diferencias. Por ejemplo, si alguno de los participantes en la interacción presenta una marca corporal visible - una deformidad, una amputación o incluso diferencias racialesque lo colocan en el rango de los "estigmatizados" socialmente, el atributo estigmatizador condiciona a los participantes en la interacción a gestionar la diferencia corporal, ya que ésta introduce incertidumbre en el orden de la interacción. Esto implica asumir que la confrontación con los otros involucra también expectativas corporales que condicionan la posibilidad/imposibilidad de establecer una interacción en términos de mayor o menor igualdad.

Por su parte, el proyecto de Merleau-Ponty fue ampliamente influenciado por la filosofía de Husserl, por la psicología Gestalt (Cerbone, 2006) y por el planteamiento de una fenomenología que propone pensar el mundo como algo más que una suma de objetos. Para Merleau-Ponty (1945), el mundo no es considerado un a priori, sino un lugar en donde las cosas advienen; un mundo fenomenal, pensado a partir de intencionalidades ${ }^{14}$ y asumido constitutivamente como un mundo polarizado de signos y de sentido (Dubé, 1989), en donde la objetividad del mundo es posible en la intersubjetividad (Merleau-Ponty, 1945). Merleau-Ponty sugiere, pues, que el sentido

13 Para Goffman, este concepto alude al área "cara a cara" donde dos o más individuos responden ante la presencia de otros actores sociales y alude a la interrelación entre encuentros de la interacción y su relación con las estructuraciones sociales más amplias (Roberts, 2006).

14 Recordemos que, para Husserl, la intencionalidad constituye la principal característica de la conciencia, asumiendo que la conciencia es un modo de ser, de comunicarse con el mundo y con los otros.

Sosa-Sánchez, I. A. (2021). Cuerpo, self y sociedad: una reflexión desde la fenomenología y el interaccionismo simbólico. Iberoforum. Revista de Ciencias Sociales, Nueva Época, 1(2), 1-18, Ensayos, e000162. https://doi.org/10.48102/if.2021.v1.n2.162 Licencia Pública Internacional - CC BY-NC-ND 4.0 
de la reducción fenomenológica debe manifestar la apertura al mundo y a la intersubjetividad (Arias, 1975).

En cuanto a la conciencia, Merleau-Ponty la aborda de manera novedosa al rechazar el dualismo cartesiano y anticiparse al proyecto desestabilizador del pensamiento binario cartesiano, presente a lo largo de la historia de la filosofía occidental (Grosz, 1993). Este autor no ubica a la conciencia al interior de la persona ni la considera perteneciente a un ámbito privado, sino que estima que se accede al conocimiento del mundo y de la conciencia a partir de las acciones e interacciones establecidas con otros actores sociales. Este abordaje alega que la acción social es intencional, es decir, dirigida y orientada por y hacia otros, hacia el mundo en el que se está inserto, y que se caracteriza por ser prerreflexiva y pragmática. Se asume, pues, que los actores sociales interactúan y experimentan el mundo socialmente, lo que significa cuestionar la existencia de un self unitario, preexistente y no socialmente constituido (Matthews, 2002).

Merleau-Ponty señala que la experiencia no puede ser considerada fuera de las relaciones sociales, políticas, culturales e históricas en las que dicha experiencia se construye (Matthews, 2002). Este autor desarrolla el concepto de Husserl de experiencia vivida y sugiere que ésta es una manera en la que, en tanto seres incorporados, nos proyectamos en el mundo. Merleau-Ponty visibiliza y teoriza la experiencia vinculándola con el locus de la conciencia; la conceptualiza como necesariamente incorporada, corporalmente constituida y encarnada en los sujetos sociales (al igual que la subjetividad) (Grosz, 1993 y 2005). Igualmente, la noción de Merleau-Ponty de experiencia vivida implica cuestionar la experiencia como inefable o "dada", por lo que no puede ser vista como fuente de la verdad o "neutra" (Grosz, 1993 y 2005).

Por otro lado, el trabajo de Merleau-Ponty ha sido central para las elaboraciones contemporáneas en la sociología del cuerpo. Se considera que una de sus principales contribuciones a la fenomenología es justamente la del rol del cuerpo vivido, el cual es un cuerpo sujeto que está inmerso en el mundo social, asumiendo que la conciencia práctica precede a la conciencia discursiva. ${ }^{15}$ En términos generales, la fenomenología identifica el cuer-

15 Esto implica reconocer que los actores sociales están insertos en el mundo social incluso antes de interiorizar un lenguaje determinado. Sin embargo, esto no descarta que los actores sociales usan los símbolos y el lenguaje de manera reflexiva.

Sosa-Sánchez, I. A. (2021). Cuerpo, self y sociedad: una reflexión desde la fenomenología y el interaccionismo simbólico. Iberoforum. Revista de Ciencias Sociales, Nueva Época, 1(2), 1-18, Ensayos, e000162. https://doi.org/10.48102/if.2021.v1.n2.162 Licencia Pública Internacional - CC BY-NC-ND 4.0 
po (más que las estructuras sociales) como la condición de la experiencia, de la conciencia y de las orientaciones de éstas hacia el mundo. Se insiste así en rechazar la separación cartesiana mente/cuerpo, pues se señala que el cuerpo es el medio de toda percepción (Shilling, 2005 y 2004; Grosz, 1993 y 2005; Cerbone, 2006).

Así, podemos afirmar que la fenomenalidad del mundo adquiere su inteligibilidad por la descripción fenomenológica que Merleau-Ponty hace del cuerpo, a partir de su reflexión en torno a la percepción, ${ }^{16}$ asumiendo que el mundo es fenomenal porque el cuerpo también lo es, lo que niega el dualismo cartesiano. Es decir, desde la perspectiva de Merleau-Ponty, se sugiere que el cuerpo es un sujeto, un "espacio" de subjetividad y conciencia. Así, el cuerpo constituye un "vehículo" activo del estar en el mundo desde una experiencia situada que se manifiesta por intencionalidades (Shilling, 2005). ${ }^{17}$ De esta manera, el cuerpo ha integrado al mundo para acceder a él; tomamos conciencia de él a partir de un esquema corporal (que es un modo de expresar que el cuerpo está en el mundo) en el que la percepción es un modo de estar en el mundo, una forma de intencionalidad (Dubé, 1989; Arias, 1975).

Se sugiere así, desde la fenomenología, que la estructura, el significado del mundo y la integridad de los objetos son llevados a cabo a través de la experiencia corporal (Shilling, 2005). Los actores sociales, según esta perspectiva, desarrollan una orientación no sólo a partir de la existencia de regímenes discursivos o gubernamentales, sino a partir de arreglos prácticos derivados de los contextos situacionales e intencionalidades que se desprenden de su existencia incorporada (Shilling, 2005).

A su vez, a partir de la reflexión de Merleau-Ponty sobre el cuerpo como sujeto, se considera que éste transforma las técnicas corporales adquiridas culturalmente en competencias y en acciones sociales efectivas. Este autor evidencia cómo este proceso es llevado a cabo a través de la conciencia y la percepción de las circunstancias. Para Merleau-Ponty

16 En Merleau-Ponty, la percepción es la actividad necesaria de un "espíritu encarnado". De esta forma, una discusión sobre el cuerpo involucra el estudio de la percepción en todas sus manifestaciones, pues considera que la percepción sintetiza nuestra relación con el mundo (Grosz, 2005 y 1993).

17 El cuerpo fenomenal en tanto "estar en el mundo" se considera como una "situación" o "existencia". Existir o ser una situación significa que el cuerpo tiene un punto de vista, una perspectiva sobre el mundo, sobre el estar inserto en el mundo (Dubé, 1989).

Sosa-Sánchez, I. A. (2021). Cuerpo, self y sociedad: una reflexión desde la fenomenología y el interaccionismo simbólico. Iberoforum. Revista de Ciencias Sociales, Nueva Época, 1(2), 1-18, Ensayos, e000162. https://doi.org/10.48102/if.2021.v1.n2.162 Licencia Pública Internacional - CC BY-NC-ND 4.0 
(1945), el campo de la percepción y el de la acción están articulados y funcionan de una manera mutua y transformadora (Crossley, 1996). Desde este enfoque, el cuerpo deviene un nudo de estructura y acción.

Lo antes mencionado obliga a problematizar y a reflexionar de maneras diferentes el self; en este proyecto, han jugado un papel central el interaccionismo (Crossley, 1996) y la fenomenología. Por ejemplo, Crossley sugiere que la obra de Goffman es medular para entender dos conceptos centrales de la teoría social sobre el cuerpo: 1) las técnicas corporales de Marcel Mauss y 2) la intercorporalidad merleaupontiana. ${ }^{18} \mathrm{Al}$ respecto, Crossley (1996) propone la interdependencia y constitución relacional de estos conceptos, asumiendo que el trabajo de Goffman constituye un ejemplo paradigmático fundado en una praxis social incorporada, así como una forma de análisis social basado en lo que Merleau-Ponty conceptualiza como la "intersubjetividad carnal" o como la "sociología carnal". ${ }^{19}$

Crossley considera que Goffman y Merleau-Ponty conceptualizan la acción social como incorporada y relacionada con el campo perceptivo del actor social (involucrando aspectos olfativos, táctiles, visuales, etcétera) (Crossley, 1996; Shilling, 2005). ${ }^{20}$ Así, las percepciones orientan la acción social hacia el "orden social de la interacción", en donde juegan un papel central el tiempo y el espacio (Dubé, 1989). ${ }^{21}$ Por ejemplo, respecto al espacio, Goffman (1974) desarrolló nociones tales como "frente" y "atrás" para dar idea de los cuadros espaciales que aluden a distintos niveles de análisis: desde el mundo físico - en donde se comparte la conciencia de los otroshasta los cuadros institucionales. En cambio, para Merleau-Ponty (1945), la percepción del espacio y la percepción de las cosas (la espacialidad de la cosa y su ser de cosa) no pueden diferenciarse. Se considera que existe una

18 La intercorporalidad o intercorporeidad se define como una extensión de los vínculos internos con el propio cuerpo (Merleau-Ponty, 1945).

19 Shilling (2005) indica que la sociología "carnal" de Merleau-Ponty opera sobre el supuesto de que el self, la sociedad y el orden simbólico se constituyen a través del cuerpo vivido (lived body).

20 Siguiendo a Goffman, Crossley (1996) sugiere que las figuras en el campo perceptivo del actor social son frecuentemente situaciones sociales que demandan adaptaciones rituales y prácticas específicas.

21 Crossley (1996) considera que Merleau-Ponty es uno de los teóricos principales de la espacialidad de la incorporación, y que es Goffman quien aporta una de las discusiones más acabadas respecto a las exigencias situacionales y territoriales a las que se enfrenta el actor social incorporado. 
reciprocidad entre el cuerpo y el mundo, a tal punto que no pueden ser diferenciados. Así, la experiencia corporal se enraíza en el espacio de la existencia, en donde "ser cuerpo" es estar vinculado al mundo, y el cuerpo no está en el espacio, sino que forma parte del mismo (Merleau-Ponty, 1945), puesto que el cuerpo espacializa al mundo en y por sus gestos (Dubé, 1989). ${ }^{22}$

Por su parte, para Goffman, no es posible discutir las técnicas corporales dejando de lado las negociaciones espaciales rutinarias y su mediación a través del orden social establecido durante la interacción (Crossley, 1996). Esta perspectiva considera que la interacción no es solamente verbal, sino que implica un simbolismo corporal que participa de un orden simbólico y en donde el intercambio de sentido se realiza a través de signos emitidos a nivel del lenguaje y del cuerpo (Le Breton, 2004). De tal suerte que se puede considerar que una de las principales aportaciones de Goffman al estudio del cuerpo es su insistente preocupación para darle realidad analítica a la copresencia corporal que estructura el vínculo social desde las respuestas recíprocas que se dan los individuos en las interacciones sociales.

Finalmente, en lo referente a la intersubjetividad, Goffman (1963) entiende la orientación de la acción y las técnicas corporales como intersubjetivas y orientadas desde y hacia los otros; subraya así la importancia de los fundamentos corporales de la experiencia de los actores sociales (las formas corporales de la interacción). Por su parte, para Merleau-Ponty, la intersubjetividad es una estructura intencional de la vida que revela el sí mismo en "situación", ${ }^{23}$ asumiéndose que la subjetividad está siempre encarnada y ligada al cuerpo (y por tanto al mundo), lo que significa hablar de un cuerpo y un mundo ontológico a la constitución del sujeto.

\section{Reflexión final: aportaciones del interaccionismo simbólico y de la fenomenología para la comprensión de las relaciones entre el cuerpo, lo social y el self}

Queremos enfatizar, como se ha visto en este ensayo, de qué manera los abordajes teóricos y conceptuales que se revisaron contribuyen de manera significativa a comprender los vínculos entre el cuerpo, lo social y el self. En

22 La noción de "carne del mundo" (chair du monde) significa que el mundo y el cuerpo están hechos recíprocamente de la misma carne (Merleau-Ponty, 1945; Grosz, 2005).

23 Para Crossley (1996), la intercorporalidad en Merleau-Ponty es la versión filosófica de lo que sociológicamente Goffman denomina el contexto del ejercicio de las técnicas corporales.

Sosa-Sánchez, I. A. (2021). Cuerpo, self y sociedad: una reflexión desde la fenomenología y el interaccionismo simbólico. Iberoforum. Revista de Ciencias Sociales, Nueva Época, 1(2), 1-18, Ensayos, e000162. https://doi.org/10.48102/if.2021.v1.n2.162 Licencia Pública Internacional - CC BY-NC-ND 4.0 
los tres abordajes analizados, podemos encontrar explícita e implícitamente conceptualizaciones que hacen referencia a la imposibilidad de pensar un self y un cuerpo fuera de la historia. Esto, si bien fue planteado ya desde la tradición interaccionista, es fenomenológicamente problematizado por Merleau-Ponty, quien considera la conciencia como consecuencia de la intencionalidad, lo que implica una conceptualización del self incorporado, es decir, un self y un cuerpo relacionales que existen sólo en relación al mundo $\mathrm{y}$ al contexto que los trasciende.

Podemos afirmar que una de las principales aportaciones desde la fenomenología (sobre todo la de Merleau-Ponty) a la reflexión que nos ocupa es haber dirigido la atención hacia la importancia del cuerpo como un vínculo entre el self y la sociedad, así como haber destacado las bases corporales de la experiencia y de la subjetividad (Shilling, 2005; Arias, 1975). ${ }^{24}$ Es decir, al plantear un sentido fenomenológico del cuerpo, enfatizando su actividad significante, el cuerpo es conceptualizado y asumido como un nudo de significaciones vivientes (Merleau-Ponty, 1945), contribuyendo a pensar de manera diferente los vínculos entre el self y el cuerpo y la relación del self con el mundo; se propone, además, un self dinámico e histórico (Matthews, 2002).

Al subrayar el carácter incorporado del self, Merleau-Ponty contribuye a visibilizar lo que Schütz, desde la sociología fenomenológica, denomina la sedimentación de la experiencia (siempre social), la cual es intersubjetiva, es decir, abierta a un mundo socialmente construido y compartido, en el cual los actores sociales atribuyen significados cultural e históricamente situados, interpretando y participando activamente así en la construcción social de la realidad con sus interacciones cotidianas (Le Breton, 2004).

En este punto, el postulado de la interpretación subjetiva aportado por Schütz (1987 y 1962) resulta central para aprehender, a través del discurso y del lenguaje de los actores sociales, la lógica social ${ }^{25}$ que articula éstos y a través de los cuales se expresa su subjetividad. Así, el lenguaje y la comunicación (verbal y no verbal), además de estar socialmente con-

24 La subjetividad carnal en Merleau-Ponty hace explícita la espacialidad y motricidad del cuerpo que se nos presenta en la noción de esquema corporal; también hace posible la percepción (Arias, 1975).

25 Conceptualizar esta lógica como social significa que es socialmente construida, es decir, con vinculaciones claras con los arreglos sociales del contexto social. 
dicionados, implican necesariamente un vínculo entre el self y su entorno social (los otros selves con los que se comunica) (Matthews, 2002). Resulta pertinente resaltar que, aunque el mismo Merleau-Ponty señalaba la importancia que juegan el tiempo y el espacio en la experiencia incorporada, esta perspectiva se vio enriquecida por el énfasis en el contexto y la situación biográfica aportados por el interaccionismo y por las reflexiones de Schütz.

Adicionalmente, la articulación de las aproximaciones abordadas al asumir el cuerpo como vehículo de la comunicación ${ }^{26}$ y del lenguaje implica que el cuerpo presenta la característica fundamental que establece la intersubjetividad: es inherentemente social. Por lo tanto, el cuerpo, en tanto objeto de estudio sociológico, puede y debe ser pensado como un nudo de estructura y acción a través del cual es posible acceder al análisis de los vínculos y relaciones entre sujeto, cuerpo y sociedad (Esteban, 2004). Esto significa reconocer que la acción social es necesariamente incorporada (Shilling, 2005). También es preciso señalar que los vínculos entre cuerpo, self y sociedad abordados en este ensayo obligan (como pudo observarse) a repensar los vínculos entre lo macro y lo microsocial y a cuestionar el pensamiento binario que ha dominado las ciencias sociales. Esto implicaría conceptualizar a los actores sociales como agentes con capacidad de interpretar activamente sus circunstancias y de adaptarse a ellas y, al mismo tiempo, reconocer que están insertos en un orden social que los moldea. Lo antes mencionado, para el objeto que nos ocupa en este ensayo, significa reconocer que los procesos macrosociales estructuran, a través de los contextos locales, la relación entre la experiencia subjetiva del cuerpo con la sociedad y viceversa.

Finalmente, es importante subrayar que las aportaciones de los abordajes presentados en este ensayo han hecho posibles muchos de los debates que abordan el estudio del cuerpo suscitados a través del tiempo en la teoría social, especialmente, pero no exclusivamente, entre autoras feministas (Adelman y Ruggi, 2013 ). Para éstas, el desmantelamiento de la oposición entre mente y cuerpo, así como la problematización e historización de los cuerpos y de las desigualdades de género, han sido elementos fundamentales de combate teórico y político. Resaltemos pues que, en

26 Lo que es presentado explícita e implícitamente en los abordajes interaccionistas, en la fenomenología de Merleau-Ponty y en la sociología fenomenológica de Schütz. Por ejemplo, para Merleau-Ponty, esa comunicación básica es siempre carnal (Arias, 1975).

Sosa-Sánchez, I. A. (2021). Cuerpo, self y sociedad: una reflexión desde la fenomenología y el interaccionismo simbólico. Iberoforum. Revista de Ciencias Sociales, Nueva Época, 1(2), 1-18, Ensayos, e000162. https://doi.org/10.48102/if.2021.v1.n2.162 Licencia Pública Internacional - CC BY-NC-ND 4.0 
algunas teóricas contemporáneas feministas interesadas en la teoría social sobre el cuerpo - como Elisabeth Grosz e Iris Marion Young-, existe un diálogo importante con las aportaciones antes presentadas, por lo que podemos afirmar que, sin éstas, algunos de los debates actuales en teoría social del cuerpo, como son los relativos a la corporalidad y al cuerpo vivido, hubieran sido difícilmente posibles.

\section{Referencias bibliográficas}

Adelman, M. y Ruggi, L. (2013). Sociología contemporánea y el cuerpo. Sociopedia, 1-16.

Allan, K. (2007). Explorations in classical sociological theory: Seeing the social world. Pine Forge Press, Thousand Oaks.

Arias, J. (1975). La antropología fenomenológica de M. Merleau-Ponty. Editorial Fragua.

Bernasconi, O. (2015). Introduciendo la moral en los estudios sociales del self. Polis. Revista Latinoamericana, (41), 1-20.

Blin, T. (1998). Introduction. En A. Schütz, Éléments de sociologie phénoménologique (pp. 4-21). L'Harmattan.

Blin, T. (2007). Introduction: Alfred Schütz et la compréhension du social. En A. Schütz, Essais sur le monde ordinaire (pp. 9-28). Le Félin/Kiro.

Cerbone, D. (2006). Understanding phenomenology. Routledge.

Crossley, N. (1996). Body techniques, agency and inter corporeality: On Goffman's relations in public. Sociology, 29(10), 133-149.

Dubé, J. (1989). Corps et monde. En L. Ponton (comp.), Séminaire MerleauPonty: Le corps-sujet (pp. 25-35). Université Laval.

Esteban, M. (2004). Antropología del cuerpo. Género, itinerarios corporales, identidad y cambio. Serie General Universitaria.

Goffman, E. (1963). Stigma. Prentice Hall.

Goffman, E. (1973). La mise en scène de la vie quotidienne. Éditions de Minuit.

Goffman, E. (1974). Les rites d'interaction. Éditions de Minuit.

Grosz, E. (1993). Merleau-Ponty and Irigaray in the flesh. Thesis eleven, (36), 37-59.

Grosz, E. (2005). Time travels: Feminism, nature, power. Duke University Press.

Le Breton, D. (2004). L'interactionnisme symbolique. Presses universitaires de France.

Matthews, E. (2002). The philosophy of Merleau-Ponty. McGill-Queen's University Press. 
Mead, H. (1962). Mind, self, E society from the standpoint of a social behaviorist. University of Chicago Press.

Merleau-Ponty, M. (1945). Phénoménologie de la perception. Gallimard.

Nizet, J. y Rigaux, N. (2005). La sociologie de Erving Goffman. La découverte.

Roberts, B. (2006). Micro social theory. Palgrave Macmillan.

Schütz, A. (1962). Collected papers. The Hague.

Schütz, A. (1987). Le chercheur et le quotidien: Phénoménologie des sciences sociales. Méridiens, Klincksieck.

Schütz, A. (1998). Éléments de sociologie phénoménologique. L'Harmattan.

Shilling, C. (2004). The body \& social theory. SAGE.

Shilling, C. (2005). The body in culture, technology and society. SAGE.

Wanderer, J. (2005). Interpretative origins of classical sociology: Weber, Husserl, Schütz, Durkheim, Simmel. Edwin Mellen Press. 